\title{
A COMPUTATIONAL APPROACH TO PRE-ALIGN POINT CLOUD DATA FOR SURFACE REGISTRATION IN IMAGE GUIDED LIVER SURGERY
}

By

\author{
Ishita Garg
}

\author{
Thesis \\ Submitted to the Faculty of the \\ Graduate School of Vanderbilt University \\ in partial fulfillment of the requirements \\ for the degree of \\ MASTER OF SCIENCE \\ in \\ Biomedical Engineering \\ December, 2007 \\ Nashville, Tennessee
}

Approved:

Dr. Robert L. Galloway

Dr. Michael I. Miga 


\section{ACKNOWLEDGEMENTS}

This works was financially supported by the Vanderbilt Institute of Integrative Biosystem Research (VIIBRE) fellowship. 


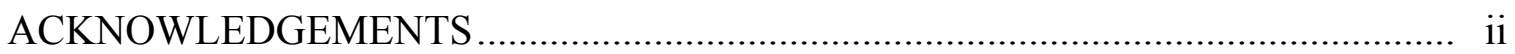

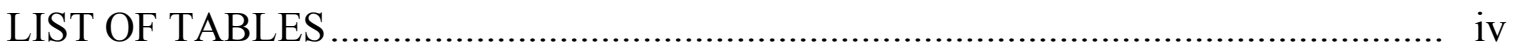

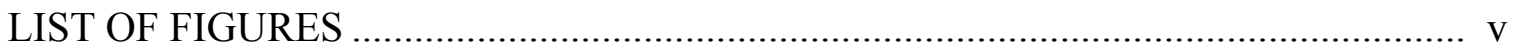

Chapter

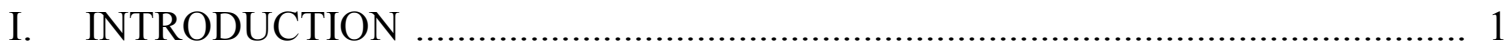

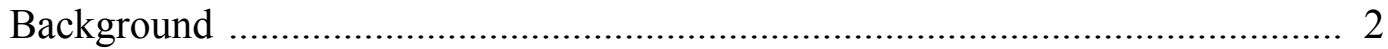

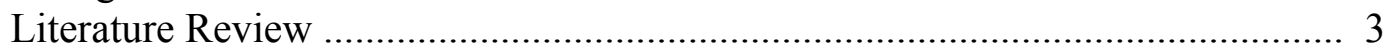

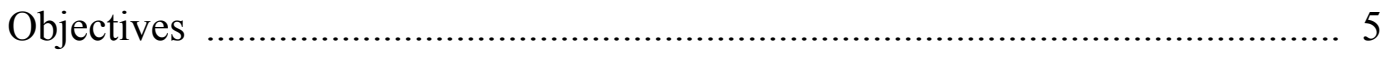

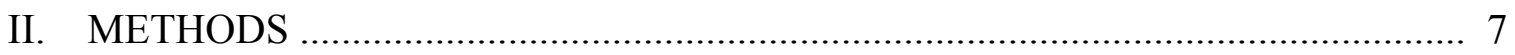

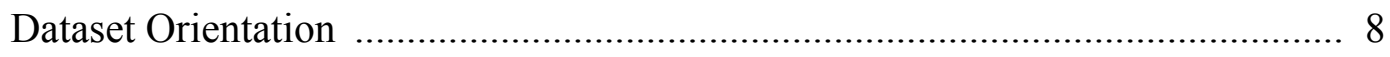

Homologous Point Computation …………………….................................... 11

Pre-operative CT Data ........................................................................ 11

Intra-operative LRS Data ................................................................... 13

Point Registration ................................................................................... 14

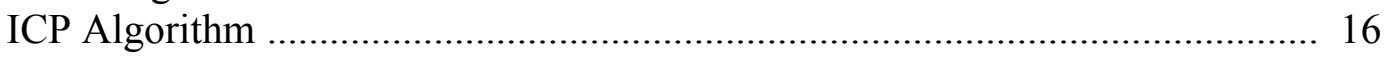

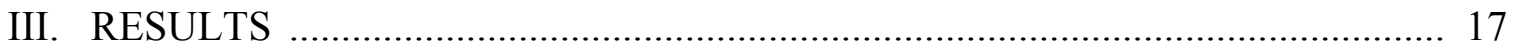

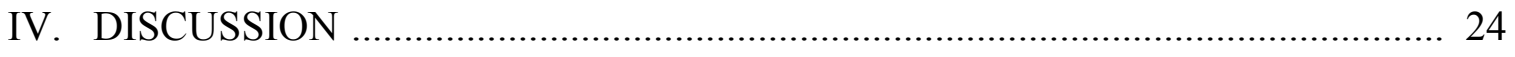

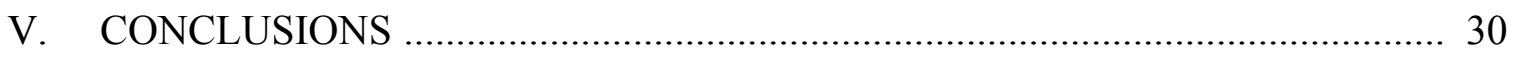

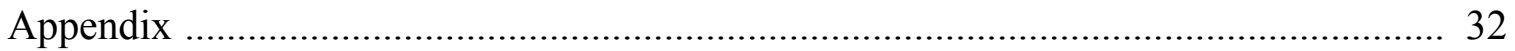

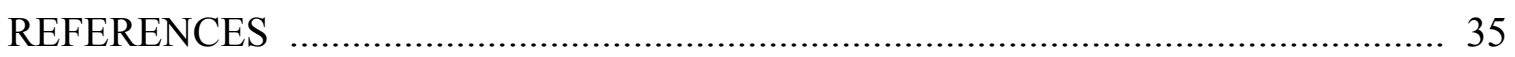




\section{LIST OF TABLES}

Table

Page

1. Comparison of the transformations for Datasets \#2 and \#4 (Figure 6) using the computational method and the anatomical landmarks method as the starting pose.

2. Effect of changing the proportion of the lengths xmax and zmax for homologous point selection on the registration results.

3. Change in the direction of vector resulting from recalculating the vector point to the extreme left of the liver to satisfy the criterion of orthogonality. The angles presented below are in degrees.

4. Effect of changing the proportion of the lengths xmax and zmax for homologous point selection on the registration results of nine datasets. ...... 


\section{LIST OF FIGURES}

Figure

Page

1. Segmented (a) pre-operative CT point cloud data and (b) intra-operative LRS point cloud data. The user selected anatomically most superior region is marked ' $\mathrm{S}$ ' and the extreme left region is marked ' $\mathrm{L}$ ' in both the cases. ..

2. Computation of the lengths (a) $\mathrm{x}_{\max }$ and (b) $\mathrm{z}_{\max }$

3. The computational cross in the $X Z$ plane containing the centroid of the demeaned data. The coordinates of the four points- $X_{1}, X_{2}, Z_{1}$, and $Z_{2}$ are shown as well.

4. Points $\mathrm{P}_{1}, \mathrm{P}_{2}, \mathrm{P}_{3}$, and $\mathrm{P}_{4}$ projected pre-operative $\mathrm{CT}$ surface data.

5. Four homologous points, $\mathrm{Q}_{1}, \mathrm{Q}_{2}, \mathrm{Q}_{3}$, and $\mathrm{Q}_{4}$ on the surface of intraoperative LRS data.

6. Comparison of the RMS residual for registration results using the computational method and anatomical landmarks to obtain the initial pose for surface-based ICP registration.

7. Comparison of the components of the translation vector: (a) $t_{x}$, (b) $t_{y}$, and (c) $t_{z}$ for the registration results using the computational method and the anatomical landmark method to obtain an initial pose for ICP algorithm. ....

8. Comparison of the Euler angles for rotation: (a) $\theta_{\mathrm{x}}$, (b) $\theta_{\mathrm{y}}$, and (c) $\theta_{\mathrm{z}}$ for the registration results using the computational method and the anatomical landmark method to obtain an initial pose for ICP algorithm.

9. Comparison of the time taken for convergence of the ICP algorithm for datasets prealigned using the computational method and the anatomical landmark method.

10. Visual comparison of the registration results of ICP algorithm using results from (a) computational method and (b) anatomical method as starting pose.

11. Misregistration seen with the (a) computational method. Better registration results with (b) anatomical method. 
12. Results of registration using (a) computational method and (b) anatomical

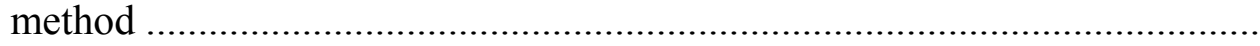

13. Registration results of the fifth dataset in Figure 6. Extraneous points due to inaccurate segmentation can be seen on extreme left of the image. 


\section{CHAPTER I}

\section{INTRODUCTION}

The need for image guided liver surgery (IGLS) arose because of the difficulty in visualizing the large vascular structures that must be avoided during hepatic surgical procedures. Fortunately, image guided neurosurgery is a more mature field and researchers have been able to translate various developments from that field to image guided liver surgery. However unique challenges in IGLS warranted the need for studies to address those specific problems. The abdominal organs exhibit a more unconstrained deformation due to factors such as gravitational forces and motion associated with respiration, unlike image guided neurosurgery where the deformation occurs in the rigid confines of the cranium. Also, unlike the cortical surface, the surface of the liver is relatively devoid of prominent anatomical features. Both of these issues make the development of a robust, accurate and fast registration method a challenging task in image guided procedures in the abdominal region. Surface registration techniques, the preferred method for IGLS, tend to require a good starting pose for convergence to a reasonable solution. Prior to this manuscript our lab has used an anatomic point based technique as a first approximation and then iterated to a solution using surface information. In this paper we present a technique which only uses the surface information to establish a starting point. 


\section{$\underline{\text { Background }}$}

Image guided surgical procedures quantitatively use information from images to guide surgical instruments. The image guided surgical system usually consists of the following components- pre-operative image acquisition, intra-operative information acquisition, registration method, and display of the intra-operative information in relation to the pre-operative images [1]. As shown in [2], there is significant liver motion associated with respiration. CT is the preferred modality for pre-operative images because of its ability to acquire in the duration of a breath hold. Various methods investigated in the past to acquire intra-operative information include intra-operative CT [3] and MRI [4], intraoperative ultrasound [5], frameless stereotaxy [2], [6] and laser range scans (LRS) [7], [8]. While intra-operative CT and MRI scans provide the greatest amount of information concerning deformation, the large expenses associated with installing these devices in the OR have prevented their widespread use. Intra-operative ultrasound images degrade after air enters the resection cavity. Image guidance techniques using surface registration, where the surface of the liver is digitized by contact with a probe have been used [2]. It was later replaced by surface acquisition using a laser range scan, which had the ability to provide denser point set information about the liver surface. Registration is the process of determining the correspondence between two spaces. In image guidance, one is the preoperative tomogram, the other the physical space. Misalignments due to poor registration compromise the quality of the image guided system. The method for establishing such correspondence may be in identifying homologous points in both spaces (image to image, image to physical); matching a point cloud from a surface in one space to a surface in another [9]; or determining some volumetric correspondence based on image identity (mutual 
information [10], [11], [12]). For matching image spaces, the first and the last methods have proved to be the most accurate and robust [13]. In matching image to physical space, only the first and second methods are possible. In abdominal soft tissue registration, point based solutions have been neither robust nor accurate, requiring the development of surface-based registration.

\section{Literature Review}

The iterative closest point (ICP) algorithm, first described in a paper by Besl and McKay in 1992, is a widely used surface registration algorithm [14]. It is a general purpose algorithm that can be applied to surfaces in various forms such as point sets, implicit and parametric curves and surfaces, and faceted surfaces. Since both the source and target data are in point set form in our study, the following description relates specifically to that data form. In the ICP algorithm, the target data is registered to the source data by iteratively finding source points that are closest to the target points. The point sets are registered using the singular value decomposition algorithm described in [15]. The final transformation resulting from the iterations is applied to the target data. The results of the ICP algorithm are highly dependent on the initial pose because the algorithm tends to find the local minima instead of the global minima.

Due to the iterative nature of most surface registration algorithms, they need a good initial alignment to avoid local minima and limit the number of iterations to the solution. The use of principal axes transformation in the context of medical image registration was described by Faber et al and Alpert et al [16, 17]. This method finds the initial alignment for the data using rigid body assumption that the position of a rigid body can be determined from 
the center of mass and rotation with respect to the center of mass. For two given sets of image data, the method assumes that the center of mass, being independent of the orientation, represents the same point in the object. The inertia matrix for the dataset can be computed in the principal axis coordinate system. The images can be registered by finding the translation from the center of mass of the system and the rotation from the inertia matrix. A major limitation of the principal axis method is its sensitivity to incomplete scan coverage $[17,18]$. In the current protocol, the liver is segmented from the CT images and the LRS images using a semi-automated segmentation technique based on the level set method [19]. In abdominal surgical procedures, externally implanted markers such as described in [20] are not feasible. In order to provide a good pose for the use of ICP algorithm, points are identified on the pre-operative images based on anatomical landmarks. Those points are then identified and digitized on the liver surface using a tracked probe and used for a point based registration to provide an initial pose for the ICP algorithm. The success of this method depends on the accurate delineation of anatomical landmarks.

Various studies in literature describe research done for the development of registration techniques and accounting for deformation. In work described in [21], a method utilizing salient anatomical features to register the data was described to make the ICP algorithm more robust to a misaligned initial pose. The method is based on a weighted geometrical features described in [22]. It uses the falciform ligament, which can be identified in both pre-operative $\mathrm{CT}$ images and intra-operative LRS images, to bias point correspondence determination. This method is dependent on the ability to extract the falciform ligament region in both the source and target data, which in turn depends on patient anatomy, image quality, and segmentation results. While most of the traditional registration 
algorithms in IGLS have been based on rigid registration algorithms, methods that account for deformations by incorporating finite element models have also been developed in the past [23].

\section{$\underline{\text { Objectives }}$}

The objective of this study is to develop a computational technique to find the initial pose for the surface-based ICP algorithm. Surface-based techniques, like all iterative registration algorithms, require a good starting pose to avoid local minima and reduce the number of iterations to the correct solution. Using anatomical landmarks to provide a good starting pose depends on individual liver anatomies. The relatively featureless surface of the liver makes it hard to localize anatomical landmarks to obtain the initial pose. Additionally the digitization process requires contact between the resected liver and the probe, potentially adding to the deformation. The principal axis transformation method, an automated technique that registers images by corresponding their principal axes, can be used for finding the initial alignment. However as mentioned before, the method is sensitive to incomplete scan coverage. Since the laser range scan is a partial representation of the liver surface, the method can not be implemented in its present form. The method presented in this manuscript is similar to the principal axis transformation method however it would require user input of the anatomical orientation of the liver. The computational method would not require contact with the probe for pre-operative and intra-operative point identification, thus reducing OR time and potential deformation. While the past studies have provided insight to improve the registration process they are still dependent on a reasonable initial pose, which currently 
requires finding anatomical landmarks. A computational method, with the above described advantages might be a valuable supplemental tool to those methods. 


\section{CHAPTER II}

\section{METHODS}

The proposed method to register intra-operative LRS images to pre-operative CT images is a semi-automated process that utilizes a priori user knowledge of anatomical orientation of the liver. Because surface registrations use iterative solutions, the process must be seeded from a location near the correct solution in order to limit the probability of converging to a local minimum. Such starting points do not require rigorous point identification, just a general orientation of two rotational directions and a translation between surfaces. Because it is easy to identify superior/inferior direction and left/right directions in both pre-operative images and laser range scan images, we have developed a technique which draws on the user's a priori ability to determine those simple orientations. The method consists of the following steps:

- Orientation of the pre-operative CT data and the intra-operative LRS data in the same direction using user input

- Computational selection of four homologous points on the surface of both the datasets

- Registration of the intra-operative LRS data to the pre-operative CT data using those four homologous points

- Use of that registration as a starting pose in the ICP surface registration algorithm. The CT data for the liver was acquired with a Siemens SOMATOM Sensation 64 CT Scanner. The liver was segmented from the tomographic image and meshed into a surface, decimated, and smoothed. The intraoperative surface data for the liver was acquired with a 
commercially available laser range scanner (Real Scan 200C, 3-D Digital Corporation, Bethel, CT). The liver was segmented from the LRS point cloud data as well. The preoperative CT point cloud data will be denoted as $\mathrm{P}=\left\{\mathrm{p}_{i}\right\}$ for $\mathrm{i}=1, \ldots, \mathrm{N}_{\mathrm{P}}$ and the intraoperative LRS point cloud data will be denoted as $Q=\left\{q_{i}\right\}$ for $i=1, \ldots N_{Q}$. The centroid of the pre-operative $\mathrm{CT}$ point cloud data, $\bar{p}$, was calculated as shown below.

$$
\bar{p}=\frac{\sum_{i=1}^{N_{P}} p_{i}}{N_{P}}
$$

where $\mathrm{N}_{P}$ is the total number of points in the CT dataset.

The centroid of the LRS dataset, $\bar{q}$, was computed similarly.

\section{Dataset Orientation}

A priori user information was incorporated into the registration process by utilizing user's knowledge of the anatomical orientation of the liver. Both the pre-operative CT and intra-operative LRS datasets were oriented in the same direction by user selection of regions anatomically corresponding to the most superior and the extreme left part of the liver as shown in Figure 1 below. 

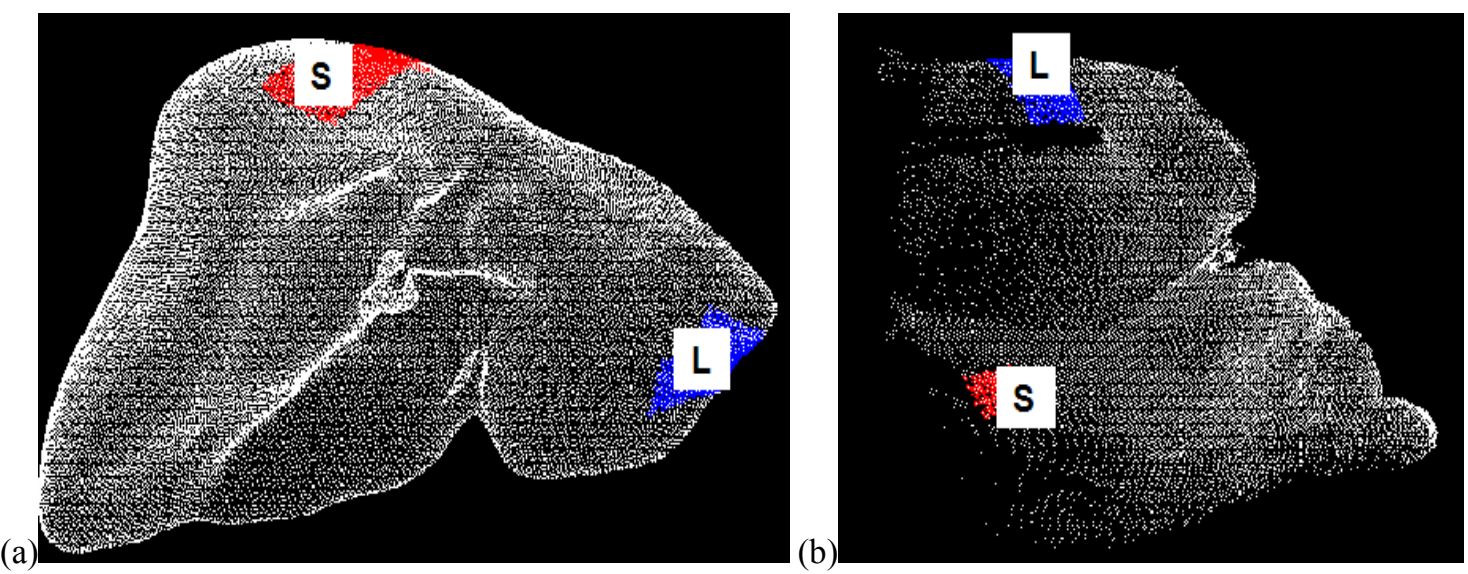

Figure 1: Segmented (a) pre-operative CT point cloud data and (b) intra-operative LRS point cloud data. The user selected anatomically most superior region is marked ' $S$ ' and the extreme left region is marked ' $L$ ' in both the cases.

Centroids of these user selected regions were calculated using equation (1) and $\bar{p}_{S}$ and $\bar{p}_{L}$ are the centroids of the most superior and the extreme left region respectively of the CT data.

Similarly $\bar{q}_{S}$ and $\bar{q}_{L}$ are the centroids of corresponding regions of the LRS data. The following unit vectors were then calculated:

$$
\begin{aligned}
& \hat{\mathrm{z}}_{p}=\frac{\bar{p}_{S}-\bar{p}}{\left|\bar{p}_{S}-\bar{p}\right|} \\
& \hat{x}_{p}^{\prime}=\frac{\bar{p}_{L}-\bar{p}}{\left|\bar{p}_{L}-\bar{p}\right|}
\end{aligned}
$$

A rotation is desired to orient the $\mathrm{CT}$ data such that $\mathrm{z}_{p}$ corresponds to the $\mathrm{z}$ axis and $x_{p}^{\prime}$ correspond to the $\mathrm{x}$ axis in a right handed coordinate system. To obtain a rotation matrix for that transformation, three orthogonal vectors are needed. The unit vectors $z_{p}$ and $\hat{x}_{p}^{\prime}$ are not necessarily perpendicular to each other. In addition a third vector is also needed for the 
rotation. In order to solve these problems the following operations were carried out. The unit vector $\hat{y}_{p}$ was calculated by finding the cross product of $\hat{z}_{p}$ and $\hat{x}_{p}^{\prime}$.

$$
\hat{y}_{p}=\hat{z}_{p} \times \hat{x}_{p}^{\prime}
$$

This would ensure that $\hat{y}_{p}$ is perpendicular to both $\hat{z}_{p}$ and $\hat{x}_{p}^{\prime}$. The unit vector pointing from the centroid of the CT dataset to the extreme left position was recalculated by a second cross product between $\hat{y}_{p}$ and $\hat{z}_{p}$.

$$
\hat{x_{p}}=\hat{y}_{p} \times \hat{z}_{p}
$$

This ensured the three unit vectors $\hat{x}_{p}, \hat{y}_{p}$, and $\hat{z}_{p}$ were orthogonal. These operations would change the direction $\hat{x}_{p}^{\prime}$ from the position originally intended by the user. The effect of this change in the direction for the vector was studied, as explained later in the results and discussion sections.

The $3 \times 3$ rotation matrix was then computed as follows:

$$
R_{p}=\left(\hat{x}_{p}, \hat{y}_{p}, \hat{z}_{p}\right)
$$

Before applying this rotation matrix to the $\mathrm{CT}$ data, it was demeaned as shown below.

$$
\tilde{p}=p-\bar{p}
$$

where $\mathrm{p}$ are the points in the CT dataset and $\bar{p}$ is centroid calculated in equation (1). It must be noted that the centroid of this demeaned $\mathrm{CT}$ dataset is zero.

$$
\frac{\sum_{i=1}^{N_{P}} \tilde{p}_{i}}{N_{P}}=0
$$


where $\mathrm{N}_{P}$ is the number of points in the CT dataset.

The rotation $\mathrm{R}_{\mathrm{p}}$ was then applied to the demeaned dataset as shown below:

$$
\tilde{p^{\prime}}=R_{p} \tilde{p}
$$

The rotation matrix to orient the LRS data, $\mathrm{R}_{\mathrm{q}}$ was calculated using a similar process as described above and it was in a similar manner to obtain analogous $\tilde{q}^{\prime}$.

\section{$\underline{\text { Homologous Points Computation }}$}

After orienting both the datasets in the same direction, four homologous points were computed on the surface of each of the datasets.

\section{Pre-operative CT Data}

The maximum lengths for the $\mathrm{CT}$ data in the $\mathrm{x}$ and $\mathrm{z}$ directions $\left(\mathrm{x}_{\max }\right.$ and $\left.\mathrm{z}_{\max }\right)$ were computed as shown in Figure 2 below.

(a)

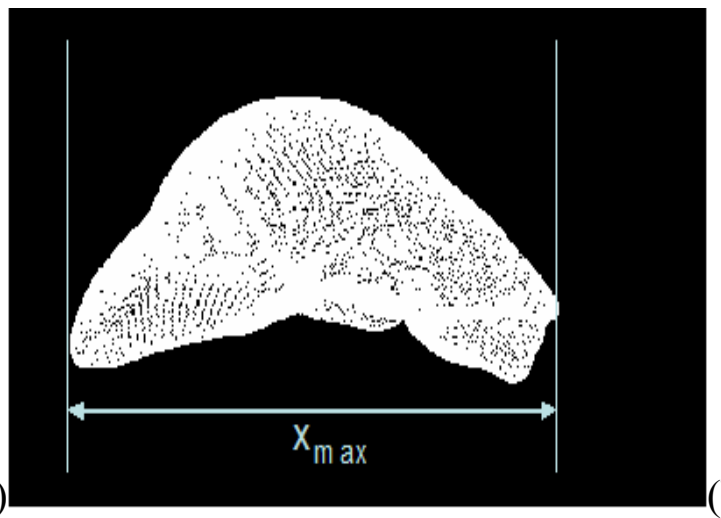

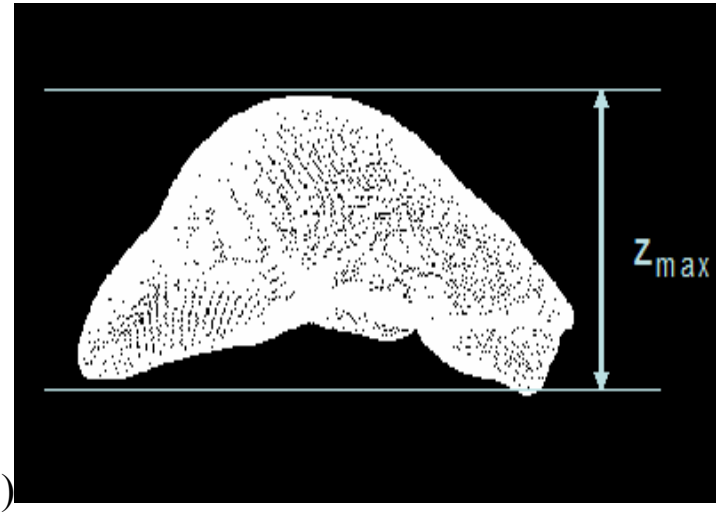

Figure 2: Computation of the lengths (a) $x_{\max }$ and (b) $z_{\max }$. 
A computational cross was then constructed in the $\mathrm{XZ}$ plane containing the centroid of the transformed CT data $\left\{\tilde{p}_{i}\right\}_{i=1}^{N}$, which is the origin as explained in equation (8) above. A certain percentage of the lengths $\mathrm{x}_{\max }$, and $\mathrm{z}_{\max }$ was used for this computational cross. Because we know that the CT scan spans all dimensions of the liver and the LRS may not, we operate on a reduced span of the CT scan. For this data, $70 \%$ of these lengths $\left(\mathrm{x}^{\prime}{ }_{\text {max }}\right.$ and $z_{\text {max }}^{\prime}$ ) were used. A retrospective study was performed to test the effects of using different proportions of the lengths $\mathrm{x}_{\max }$ and $\mathrm{z}_{\max }$ on the overall registration results, which are described in the Results and Discussion section. Four points, $X_{1}, X_{2}, Z_{1}$, and $Z_{2}$ were calculated on the computational cross as shown in Figure 3 below.

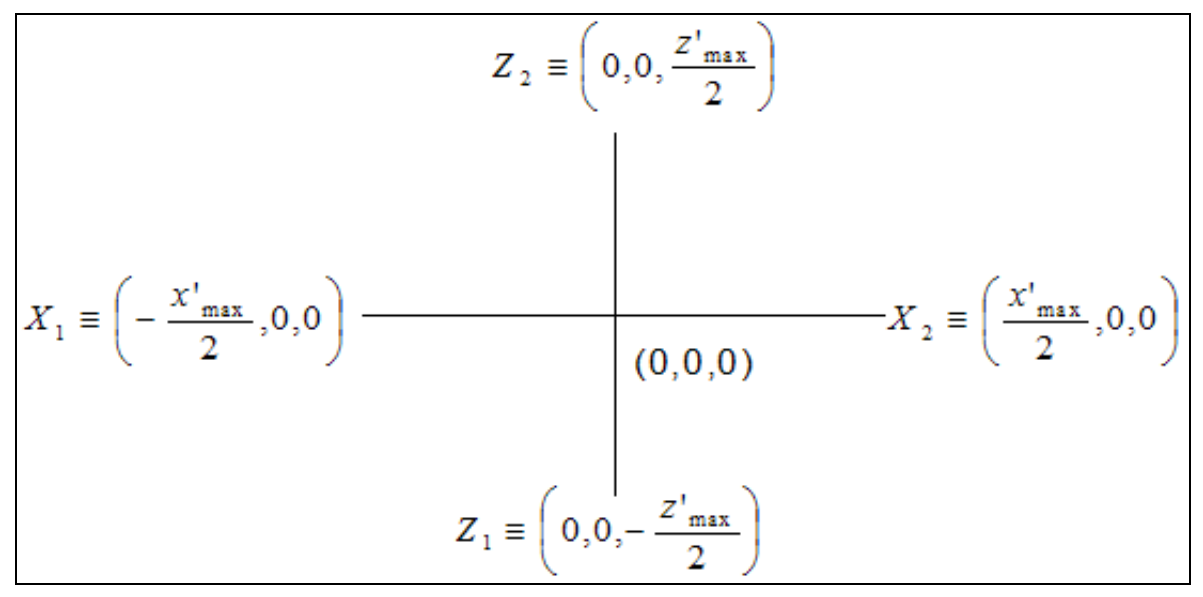

Figure 3: The computational cross in the $X Z$ plane containing the centroid of the demeaned data. The coordinates of the four points- $X_{1}, X_{2}, Z_{1}$, and $Z_{2}$ are shown as well.

These four points were projected onto the anterior surface of the CT dataset as shown in

Figure 4. 


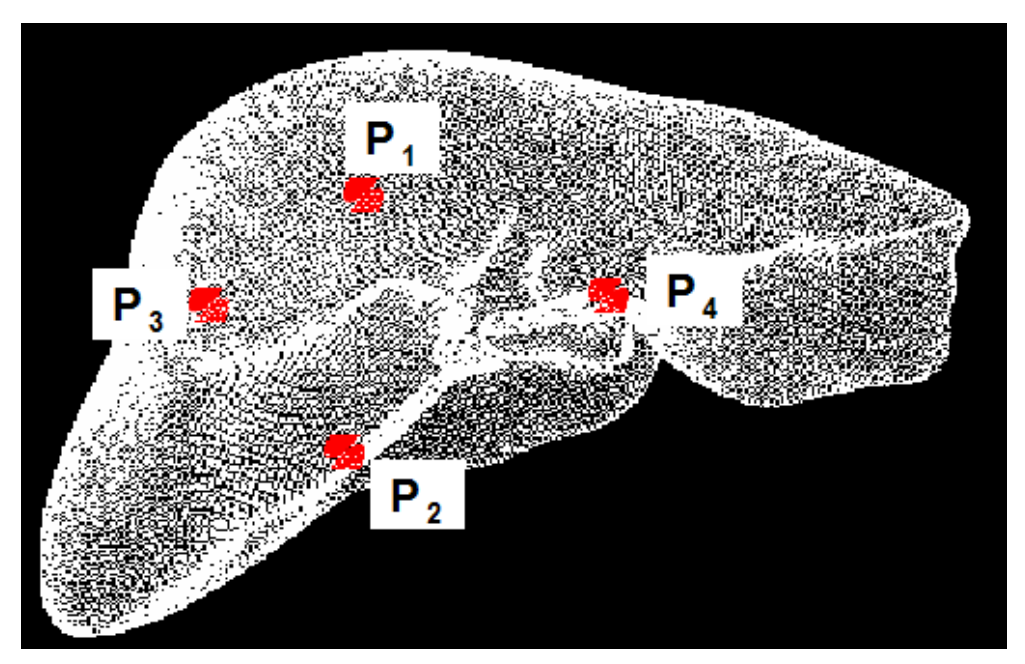

Figure 4: Points $P_{1}, P_{2}, P_{3}$, and $P_{4}$ projected pre-operative $C T$ surface data.

\section{Intra-operative LRS Data}

The four homologous points for the LRS data were found using a similar computational cross. The distances $\mathrm{x}^{\prime}{ }_{\max }$ and $\mathrm{z}_{\text {max }}{ }_{\text {ax }}$ were conserved for the LRS data and after accounting for the scaling, four points, $\mathrm{Q}_{1}, \mathrm{Q}_{2}, \mathrm{Q}_{3}$, and $\mathrm{Q}_{4}$ were obtained by using the same method as described for the CT data. The points projected on the LRS data are shown in Figure 5 below.

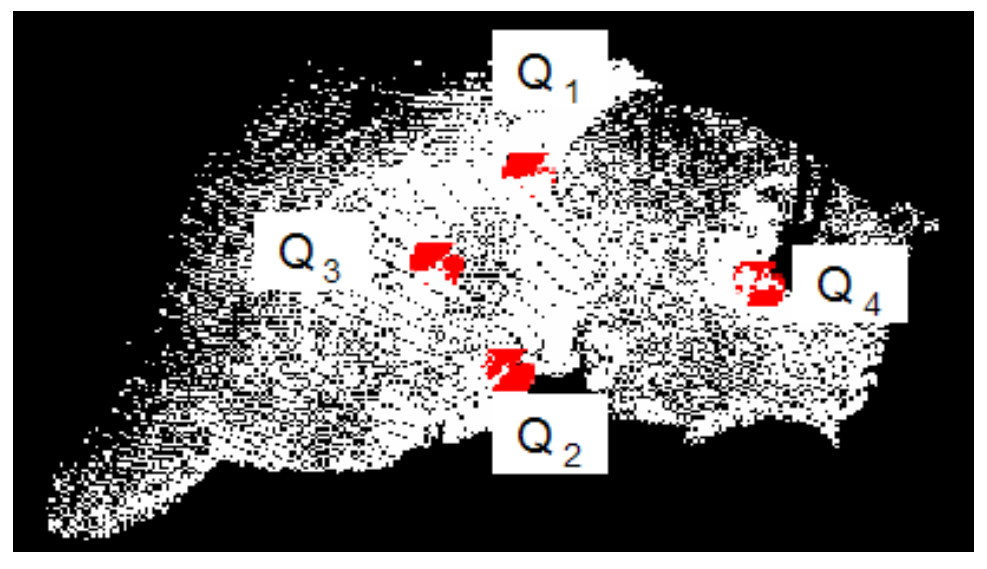

Figure 5: Four homologous points, $Q_{1}, Q_{2}, Q_{3}$, and $Q_{4}$ on the surface of intra-operative LRS data. For the point registration process, the points $\mathrm{Q}_{1}$ through $\mathrm{Q}_{4}$ are considered homologous to points $\mathrm{P}_{1}$ through $\mathrm{P}_{4}$ because both datasets were oriented in the same direction. These four 
points were used to compute a point registration between the two datasets as described in the next section.

\section{$\underline{\text { Point Registration }}$}

The four homologous points were used to register the LRS dataset to the CT dataset using singular value decomposition method [15] to obtain a translation vector $\mathbf{t}$ and a rotation matrix $R_{q}^{\prime}$. The rotation matrix was obtained in the following manner:

$$
H=\sum_{i=1}^{4} Q_{i} \cdot P_{i}^{t}
$$

where the superscript ' $t$ ' denotes a transpose.

Then the singular value decomposition of $\mathrm{H}$ is performed as shown below:

$$
H=U \Lambda V^{t}
$$

The rotation was then found as follows:

$$
R_{q}^{\prime}=\operatorname{Vdiag}(1,1, \operatorname{det}(V U)) U^{t}
$$

The rotation $\mathrm{R}_{\mathrm{q}}$ thus obtained is a $3 \times 3$ matrix. The translation vector $\mathbf{t}$ is obtained as shown in equation (13) below.

$$
\begin{aligned}
& \mathbf{t}=\bar{P}-R_{q}^{\prime} \bar{Q} \\
& \text { where } \bar{P}=\frac{\sum_{i=1}^{4} P_{i}}{4} \\
& \text { and } \bar{Q}=\frac{\sum_{i=1}^{4} Q_{i}}{4}
\end{aligned}
$$


The goal of the registration process is to register the intra-operative LRS data to the preoperative CT data. The position in the image space is the reference and can not be changed during the registration process. The final transformation must be applied only to the LRS data. Thus, a single transform was obtained from the previously calculated transforms, $R_{p}$, $\mathrm{R}_{\mathrm{q}}, \mathrm{R}_{\mathrm{q}}$ and $\mathrm{t}$. This can be done by using the $4 \times 4$ transformation matrix format.

$$
T_{1}=\left[\begin{array}{cccc}
R_{p}(1,1) & R_{p}(1,2) & R_{p}(1,3) & 0 \\
R_{p}(2,1) & R_{p}(2,2) & R_{p}(2,3) & 0 \\
R_{p}(3,1) & R_{p}(3,2) & R_{p}(3,3) & 0 \\
0 & 0 & 0 & 1
\end{array}\right] \cdot\left[\begin{array}{cccc}
1 & 0 & 0 & -\bar{p}_{x} \\
0 & 1 & 0 & -\bar{p}_{y} \\
0 & 0 & 1 & -\bar{p}_{z} \\
0 & 0 & 0 & 1
\end{array}\right]
$$

$T_{1}$ is the transform equivalent of the demeaning process in equation (7) and the rotation $R_{p}$ in equation (9). $\bar{p}_{x}, \bar{p}_{y}$, and $\bar{p}_{z}$ are the co-ordinates of the centroid of the CT data, $\bar{p}$ calculated in equation (1). Similarly the equivalent transform $\mathrm{T}_{2}$ is obtained for the LRS data.

$$
T_{2}=\left[\begin{array}{cccc}
R_{q}(1,1) & R_{q}(1,2) & R_{q}(1,3) & 0 \\
R_{q}(2,1) & R_{q}(2,2) & R_{q}(2,3) & 0 \\
R_{q}(3,1) & R_{q}(3,2) & R_{q}(3,3) & 0 \\
0 & 0 & 0 & 1
\end{array}\right] \cdot\left[\begin{array}{cccc}
1 & 0 & 0 & -\bar{q}_{x} \\
0 & 1 & 0 & -\bar{q}_{y} \\
0 & 0 & 1 & -\bar{q}_{z} \\
0 & 0 & 0 & 1
\end{array}\right]
$$

Also,

$$
T_{3}=\left[\begin{array}{cccc}
R_{q}^{\prime}(1,1) & R_{q}^{\prime}(1,2) & R_{q}^{\prime}(1,3) & 0 \\
R_{q}^{\prime}(2,1) & R_{q}^{\prime}(2,2) & R_{q}^{\prime}(2,3) & 0 \\
R_{q}^{\prime}(3,1) & R_{q}^{\prime}(3,2) & R_{q}^{\prime}(3,3) & 0 \\
0 & 0 & 0 & 1
\end{array}\right]\left[\begin{array}{cccc}
1 & 0 & 0 & \mathbf{t}_{x} \\
0 & 1 & 0 & \mathbf{t}_{y} \\
0 & 0 & 1 & \mathbf{t}_{z} \\
0 & 0 & 0 & 1
\end{array}\right]
$$

where $\mathbf{t}_{\mathrm{x}}, \mathbf{t}_{\mathrm{x}}, \mathbf{t}_{\mathrm{x}}$ are the components of the translation vector $\mathbf{t}$ calculated in equation (13). These three transforms can be combined to a single transform. 


$$
T=T_{1}^{-1} T_{3} T_{2}
$$

The transform, $\mathrm{T}$ obtained from this method was applied to the original LRS data.

$$
q^{\prime}=T q
$$

\section{$\underline{\text { ICP algorithm }}$}

The transformed data, q' was used as a starting pose to find the transform, $\mathrm{T}_{4}$ using iterative closest point algorithm.

$$
T^{\prime}=T_{4} T
$$

The overall transformation, $\mathrm{T}$ ' obtained using the method described above was compared to the transform obtained by using anatomical points to find the starting pose for iterative closest point algorithm. Nine different datasets were tested and the results compared using each of the methods. The parameters compared were the RMS residual distances, the translation vectors, the Euler angles, and the computation times. 


\section{CHAPTER III}

\section{RESULTS}

The comparison of the RMS residuals of the nine clinical datasets obtained using the computational method and the anatomical method to obtain the initial pose for surface registration is shown in Figure 6 below.

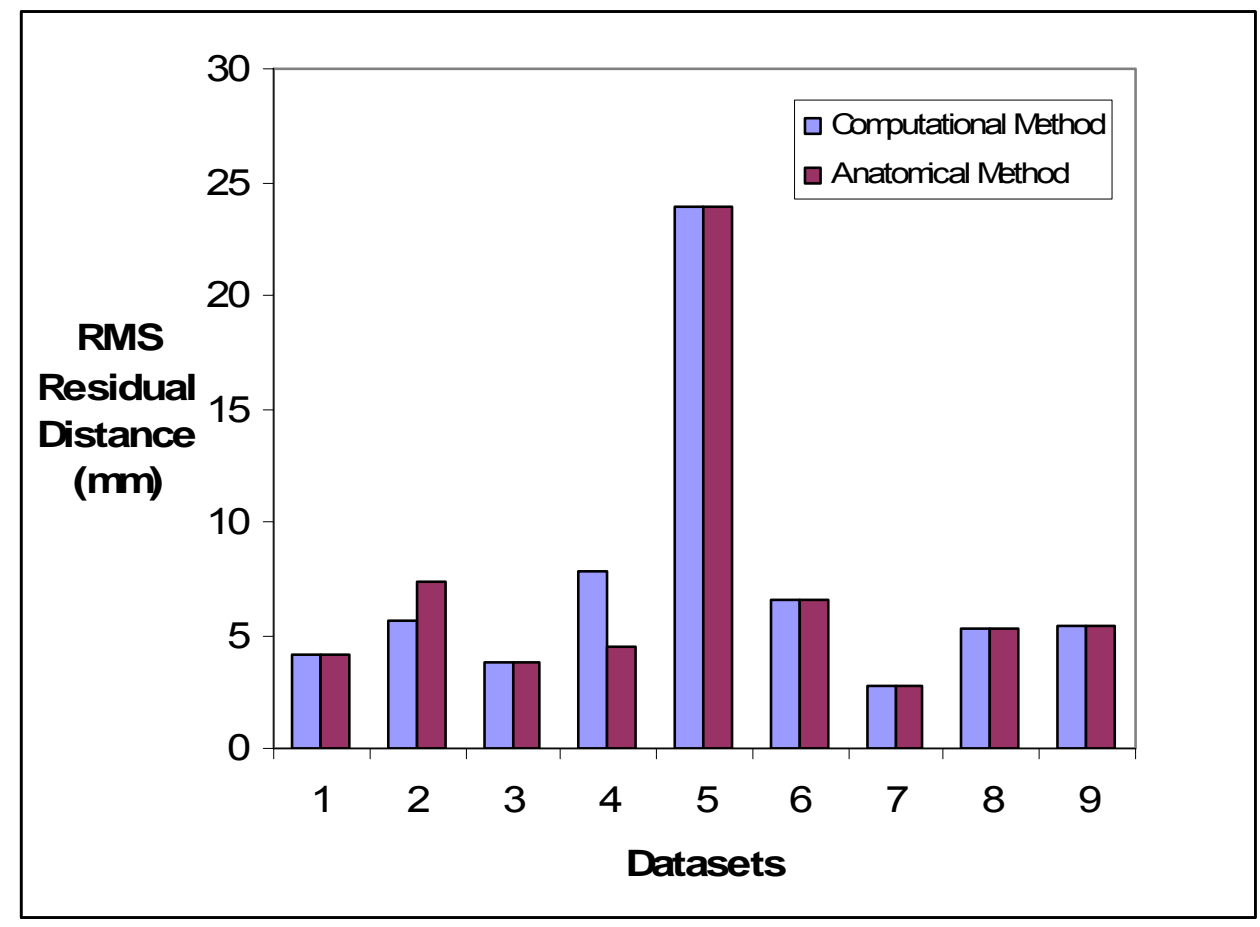

Figure 6: Comparison of the RMS residual for registration results using the computational method and anatomical landmarks to obtain the initial pose for surface-based ICP registration.

The RMS residual distance is most similar for seven of the nine datasets. The RMS residual obtained from the two methods is dissimilar in datasets two and four. Also the RMS residual is comparatively large for the fifth dataset as compared to all other datasets. The disparities in the RMS residual values were not surprising based on visual examination of the registration 
results. The second dataset did not yield satisfactory results with either registration approach because of possible organ deformation. The computational approach failed in the fourth dataset because the algorithm requires the LRS scan to acquire majority of the anterior surface of the liver, a condition that was not met in this case. The reason for the unusually large RMS residual for the fifth dataset was inaccurate segmentation. These disparities are elaborated in greater detail in the Discussion section. The mean RMS residual value for the remaining datasets using the computational method and the anatomical method was the same, $4.66 \pm 1.34 \mathrm{~mm}$. RMS residual value is a metric for error but similar values do not necessarily indicate similar registration results. In order to quantitatively evaluate the similarity of the registration results, the translations and Euler angles of rotation were compared for the two methods. Since there is a known misalignment for two of the datasets, those datasets were not included in the following analysis.

The comparison of the translation vectors for the seven clinical datasets registered with the computational and the anatomical method are shown in Figure 7 below. 

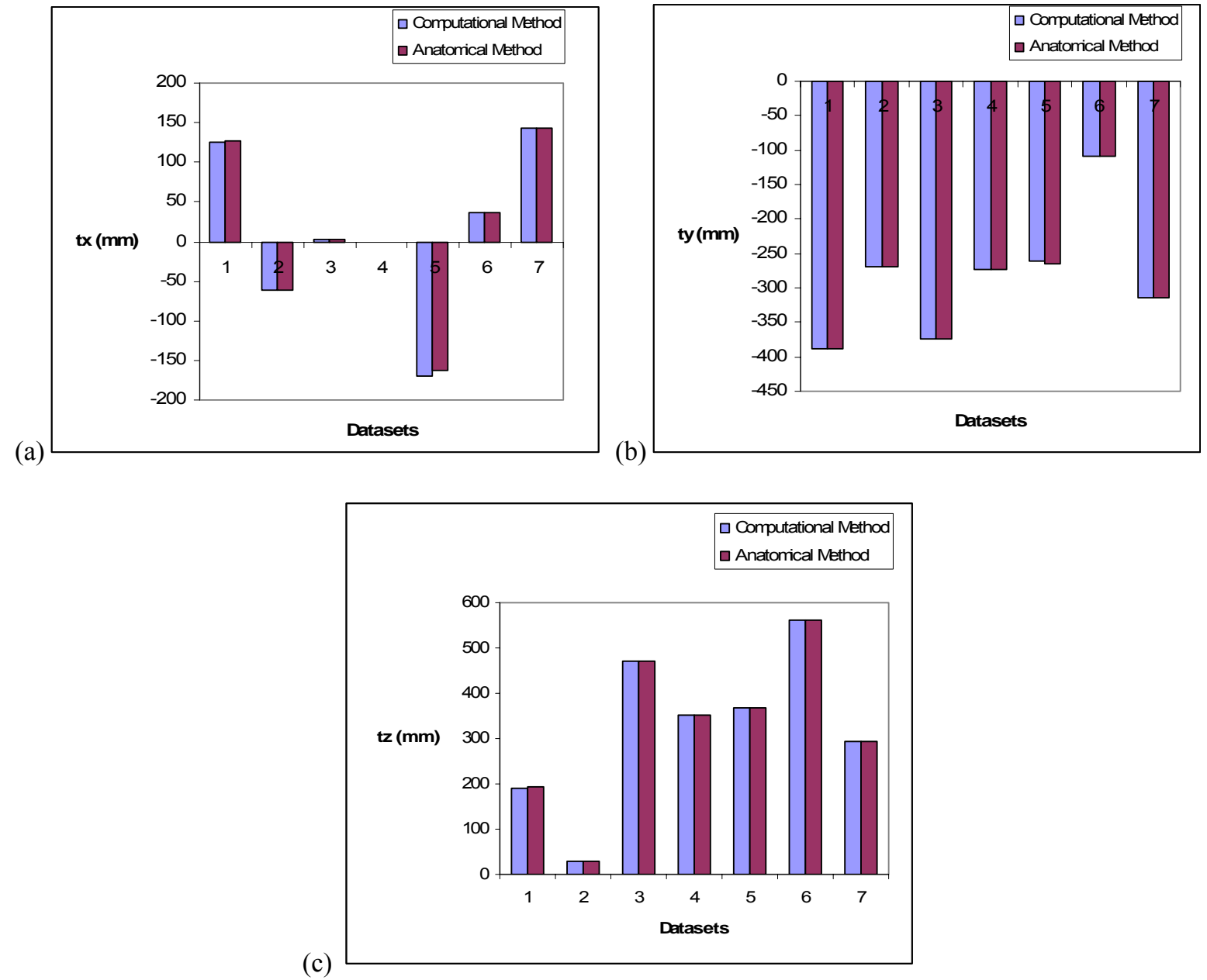

Figure 7: Comparison of the components of the translation vector: (a) $t_{x}$, (b) $t_{y}$, and (c) $t_{z}$ for the registration results using the computational method and the anatomical landmark method to obtain an initial pose for ICP algorithm.

The mean of the absolute values of the differences between the components of the translation vector obtained from the two methods are $1.11 \pm 2.25 \mathrm{~mm}, 0.67 \pm 1.54 \mathrm{~mm}$, and $0.26 \pm 0.33 \mathrm{~mm}$ for $\mathrm{t}_{\mathrm{x}}, \mathrm{t}_{\mathrm{y}}$, and $\mathrm{t}_{\mathrm{z}}$ respectively. The differences are relatively small as seen from the values presented above and the visual representation of results in Figure 7.

The comparison of the Euler angles for rotation for the seven clinical datasets registered with the computational and the anatomical method are shown in Figure 8 below. 

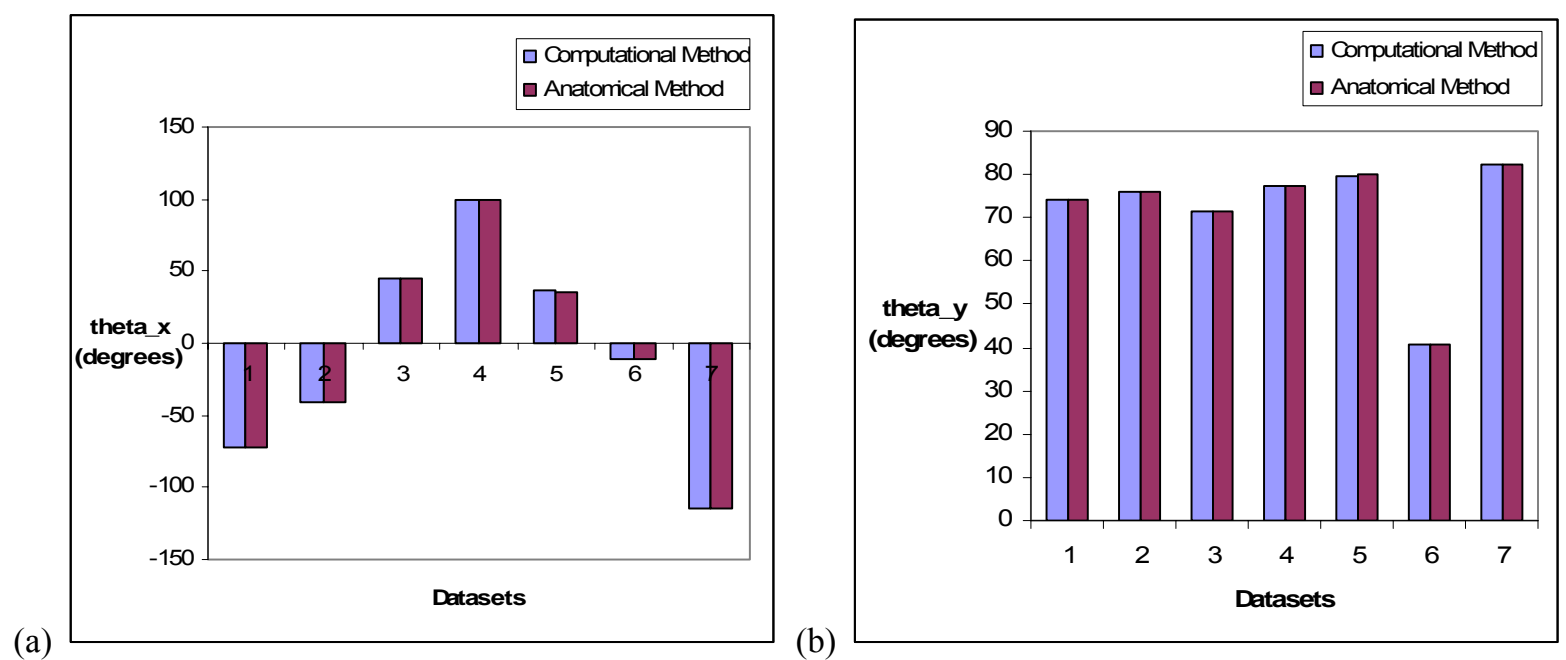

(b)

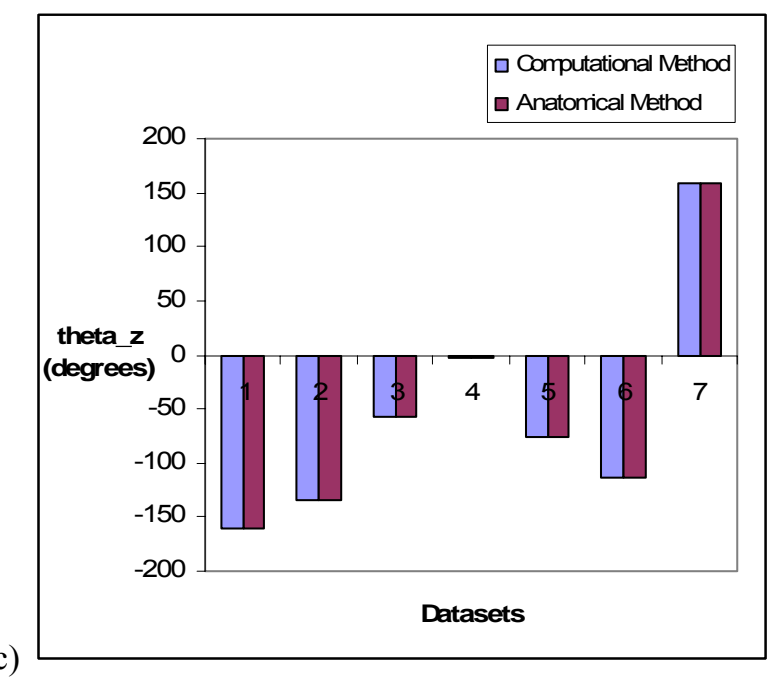

Figure 8: Comparison of the Euler angles for rotation: (a) $\theta_{x}$, (b) $\theta_{y}$, and (c) $\theta_{z}$ for the registration results using the computational method and the anatomical landmark method to obtain an initial pose for ICP algorithm.

The mean of the absolute values of differences between the Euler angles of rotation obtained from the two methods are $0.17^{\circ} \pm 0.20^{\circ}, 0.06^{\circ} \pm 0.07^{\circ}$, and $0.12^{\circ} \pm 0.13^{\circ}$ for $\theta_{\mathrm{x}}, \theta_{\mathrm{y}}$, and $\theta_{\mathrm{z}}$ respectively. From the transformation results presented above for the seven datasets, it can be concluded that the ICP algorithm converges to the same solution using the starting pose from both the computational method and the anatomical landmarks method.

The quantitative comparison of the transformations obtained for the datasets labeled two and four in Figure 6 is presented in Table 1 below. 
Table 1: Comparison of the transformations for Datasets \#2 and \#4 (Figure 6) using the computational method and the anatomical landmarks method as the starting pose.

\begin{tabular}{|c|c|c|c|c|}
\hline & \multicolumn{2}{|c|}{ Dataset \#2 } & \multicolumn{2}{c|}{ Dataset \#4 } \\
\hline Parameters & $\begin{array}{c}\text { Computational } \\
\text { Method }\end{array}$ & $\begin{array}{c}\text { Anatomical } \\
\text { Method }\end{array}$ & $\begin{array}{c}\text { Computational } \\
\text { Method }\end{array}$ & $\begin{array}{c}\text { Anatomical } \\
\text { Method }\end{array}$ \\
\hline$t_{x}$ & -133.52 & 33.26 & -288.45 & 45.05 \\
\hline$t_{y}$ & -225.38 & -332.81 & 7.72 & 75.17 \\
\hline$t_{z}$ & 304.34 & 216.73 & 428.65 & 587.95 \\
\hline$\theta_{x}$ & -67.33 & 141.41 & 7.35 & -10.00 \\
\hline$\theta_{y}$ & 59.40 & 87.90 & 79.19 & 27.46 \\
\hline$\theta_{z}$ & 159.43 & 38.69 & -145.00 & 179.75 \\
\hline
\end{tabular}

The values presented in Table 1 supports the contention based on the RMS values about the disparity in the registration results using the starting positions from the computational method and the anatomical landmarks method for those two datasets.

The comparison of the computational time for registration using the ICP algorithm for the seven clinical datasets prealigned using the computational and the anatomical landmarks method are shown in Error! Reference source not found. below.

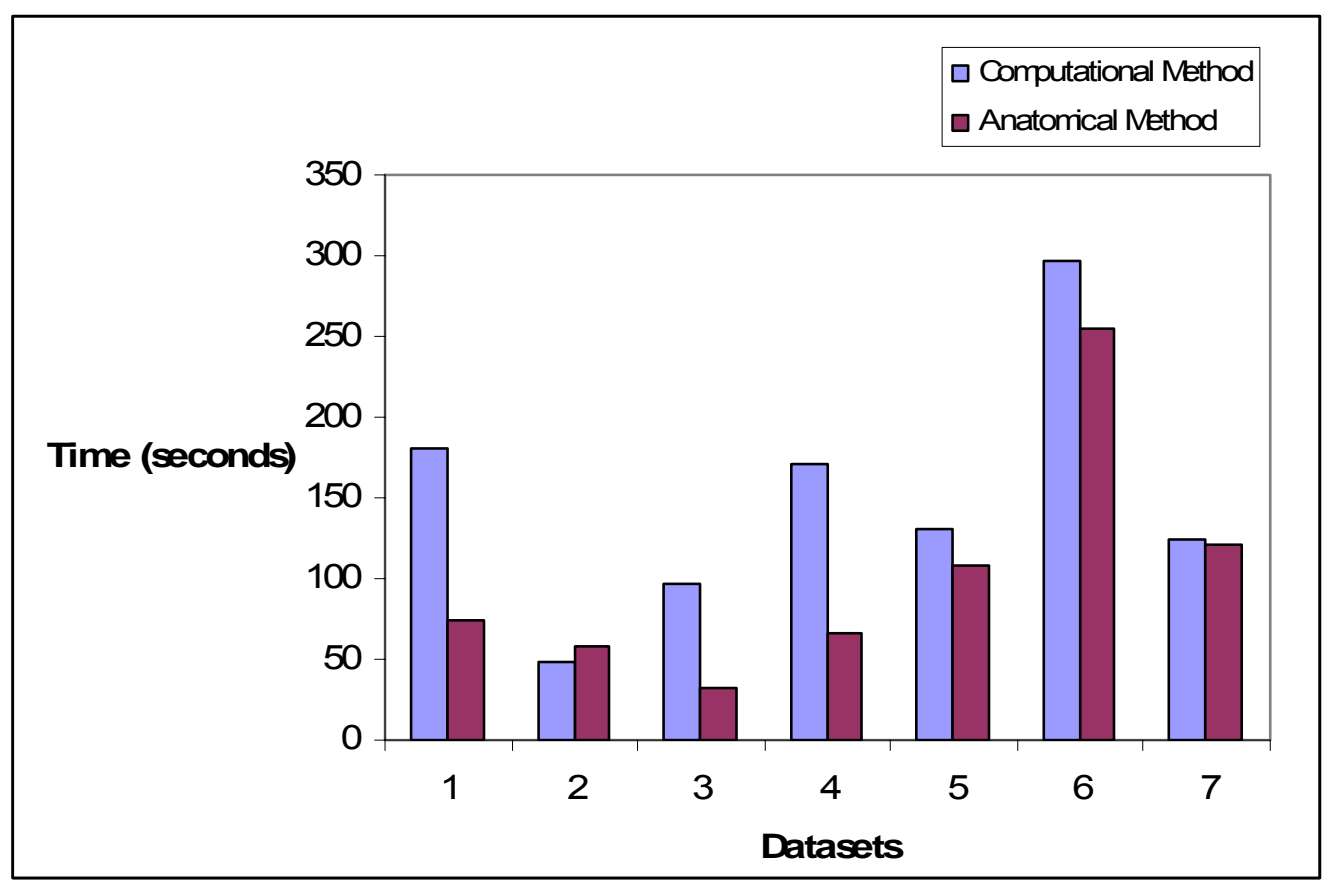

Figure 9: Comparison of the time taken for convergence of the ICP algorithm for datasets prealigned using the computational method and the anatomical landmark method. 
In most of the datasets, the ICP algorithm took longer to find the solution using the results from the computational method as the starting pose. The mean time to solution for the ICP algorithm using the results of the computational method as starting pose was $150.08 \pm 78.74 \mathrm{~s}$ and using the anatomical method as starting pose was $101.85 \pm 73.61 \mathrm{~s}$. A comparison between the computational times performed using the paired t-test statistic shows that the time differences aren't statistically significant $(\mathrm{p}<0.05)$.

The results of the retrospective studies to test some aspects of the algorithm are presented below. As mentioned previously in the Methods section, a study was performed to observe the effects of changing the proportions of the lengths $\mathrm{x}_{\max }$ and $\mathrm{z}_{\max }$ for selecting the homologous points. The effects were studied on all nine datasets and the results are presented in the appendix. Results for one of the datasets are presented in Table 2 below.

Table 2: Effect of changing the proportion of the lengths $x_{\max }$ and $z_{\max }$ for homologous point selection on the registration results.

\begin{tabular}{|c|c|c|c|c|c|c|c|c|}
\hline $\begin{array}{c}\% \text { of } \\
\text { lengths }\end{array}$ & 40.00 & 50.00 & 60.00 & 70.00 & 80.00 & 90.00 & $\begin{array}{c}\text { Standard } \\
\text { Deviation }\end{array}$ & $\begin{array}{c}\text { Anatomical } \\
\text { Method }\end{array}$ \\
\hline $\begin{array}{c}\text { RMS } \\
\text { Residual }\end{array}$ & 4.16 & 4.16 & 4.16 & 4.16 & 4.16 & 4.16 & 0.00 & 4.16 \\
\hline $\mathrm{t}_{x}$ & 44.53 & 45.36 & 108.41 & 125.72 & 2.92 & 41.11 & 46.31 & 126.30 \\
\hline $\mathrm{t}_{y}$ & -216.65 & -226.65 & -372.03 & -388.26 & -363.36 & -377.22 & 79.77 & -388.15 \\
\hline $\mathrm{t}_{z}$ & 495.26 & 484.17 & 260.81 & 191.33 & 203.49 & 163.35 & 150.58 & 192.25 \\
\hline$\theta_{x}$ & -13.10 & -13.47 & -48.49 & -72.47 & -57.40 & -80.38 & 28.79 & -72.27 \\
\hline$\theta_{y}$ & 48.57 & 50.04 & 71.84 & 74.10 & 77.79 & 76.18 & 13.42 & 73.98 \\
\hline$\theta_{z}$ & -114.42 & -114.26 & -138.72 & -160.41 & -157.45 & -176.45 & 25.65 & -160.16 \\
\hline
\end{tabular}

The results presented in each column were obtained by changing the proportion of both $\mathrm{x}_{\max }$ and $z_{\max }$ by the same percentage. The standard deviation values across all parameters indicate that the registration results are sensitive to the length of the computational cross. The registration results for the dataset using the anatomical landmarks method is also presented in the table for comparison. The registration results using the computational method as the 
starting pose are most similar to the results of anatomical landmarks method using the value of $70 \%$ of the length of the computational cross.

Lastly, for each of the nine datasets, the change in the direction of the vector denoting the extreme left of the liver (from $x^{\prime}$ to $x$ ) was also noted. These results are presented in Table 3 below.

Table 3: Change in the direction of vector resulting from recalculating the vector point to the extreme left of the liver to satisfy the criterion of orthogonality. The angles presented below are in degrees.

\begin{tabular}{|c|c|c|}
\hline Datasets & Pre-operative CT Data & $\begin{array}{c}\text { Intra-operative LRS } \\
\text { Data }\end{array}$ \\
\hline 1 & 10.35 & 19.67 \\
\hline 2 & 17.04 & 47.31 \\
\hline 3 & 4.78 & 0.08 \\
\hline 4 & 15.97 & 0.55 \\
\hline 5 & 20.68 & 15.08 \\
\hline 6 & 30.57 & 17.01 \\
\hline 7 & 2.38 & 2.50 \\
\hline 8 & 13.29 & 13.46 \\
\hline 9 & 13.33 & 1.51 \\
\hline
\end{tabular}

The order of datasets in Table 3 above corresponds to the order presented in Figure 6 . The mean change of the angle of the vector in the pre-operative CT data was $14.26 \pm 8.41^{\circ}$ and for the intra-operative LRS data the change was $13.02 \pm 15.03^{\circ}$. The mean change of the angle of the vector for seven datasets (excluding the second and the fourth) in the CT data and the LRS data were $13.62 \pm 9.60^{\circ}$ and $9.90 \pm 8.24^{\circ}$ respectively. A statistical comparison using a paired t-test statistic showed that the angle was significantly higher for the CT data $(p>0.05)$. Since there is no apparent correlation between the change in the direction of the vector and the transformations (shown in Figure 7 and Figure 8), the method is relatively insensitive to the change. 


\section{CHAPTER IV}

\section{DISCUSSION}

Accurate registration results are essential to ensure the quality of an image guided surgical system. Organ deformation and paucity of reliable anatomical landmarks on the liver surface make the development of a robust registration technique a challenge. The objective of this work was the development of a computational approach to find a reasonable the prealignment of data for surface-based ICP registration algorithm. Currently the initial alignment for surface registration is found by localizing anatomical landmarks on the surface of the liver. As the ICP algorithm can correct a reasonably approximated initial pose, the computational approach does not require precise delineation of anatomical landmarks. In addition, a computational approach would reduce contact between the liver surface and the probe for digitizing those anatomical landmarks, reducing OR time and potential deformation.

The results of the use of computational method to find the initial pose were presented for nine datasets and compared to the results from the anatomical landmarks method, using the latter as the "gold standard". RMS residual is an indicator of the similarity of the registration results. The RMS residual values, presented in Figure 6, for seven of the nine datasets were most similar, indicating that similar transformations were computed by both methods. The mean RMS residual value for the seven datasets using the computational method and the anatomical method was the same, $4.66 \pm 1.34 \mathrm{~mm}$. The comparison of the transformations obtained from the two methods (Figure 7 and Figure 8) is consistent with the 
conclusions drawn from RMS residual values. The mean difference between the translation vector components obtained from the computational method and the anatomical landmarks method for the seven datasets was fairly small. Similar results were obtained in the comparison of the Euler angles of rotation. This indicates similar registration results using the starting poses from the two methods. This conclusion was confirmed by visual inspection of the registration results. The seven datasets showed visually similar registration results for both methods. An example is presented in Figure 10 below.
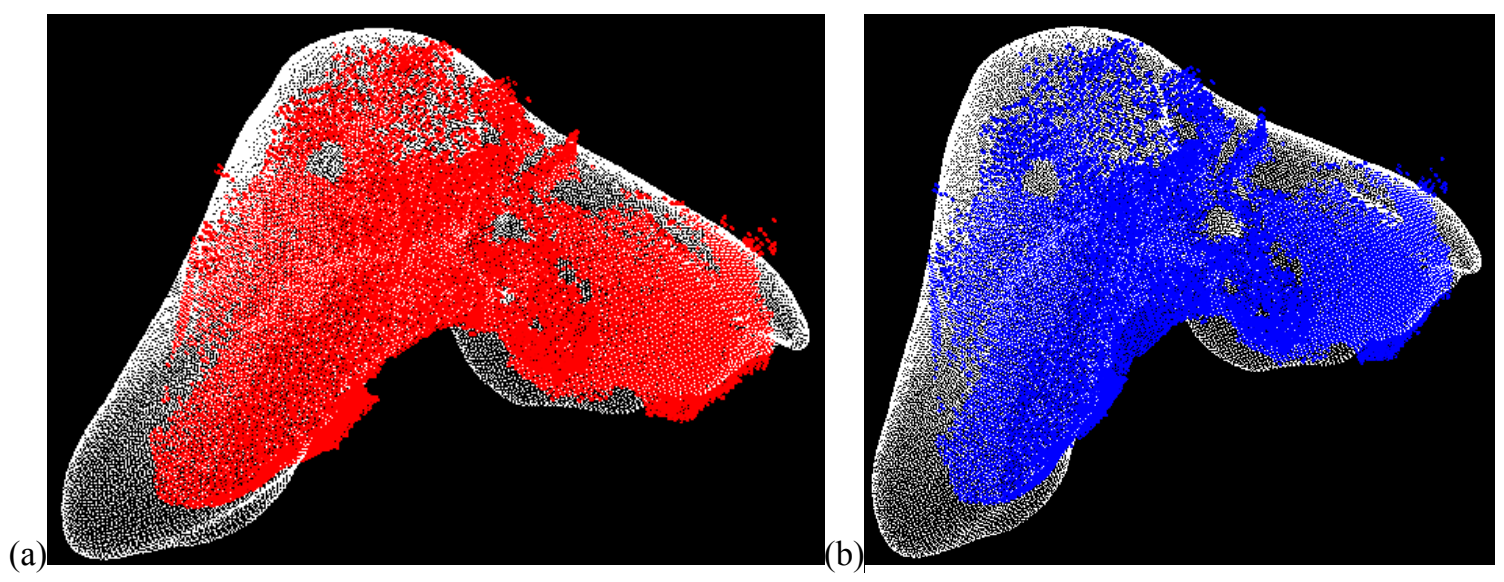

Figure 10: Visual comparison of the registration results of ICP algorithm using results from (a) computational method and (b) anatomical method as starting pose.

As seen in Figure 10 the registration results in both the cases seem well aligned. On the other hand, the visual inspection of the registration results of the two datasets presented in Table 1 showed marked difference for the two methods (Figure 11 and Figure 12). One of the limitations of the computational method is that for it to work optimally the laser range scan must cover a major portion of the anterior surface of the liver. As seen in Figure 11, for this particular dataset the laser range scan only covers a partial view of the anterior surface. 

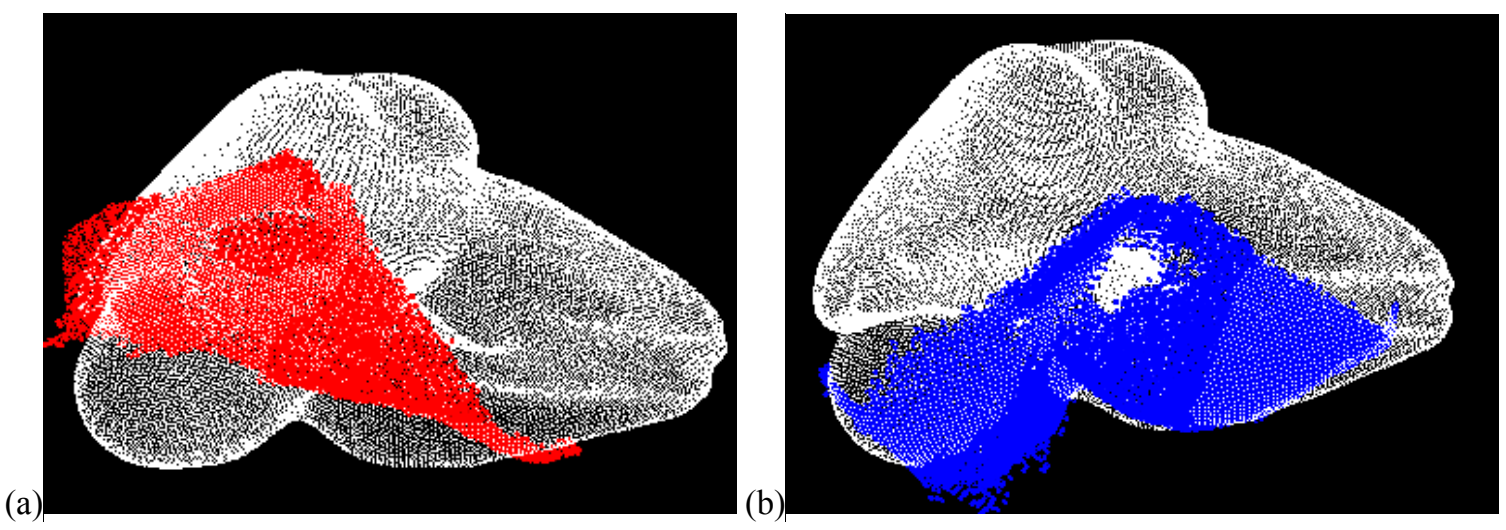

Figure 11: Misregistration seen with the (a) computational method. Better registration results with (b) anatomical method.

In the projection of the points from the computational cross (shown in Figure 3) to the surface of the CT and LRS data, various approximations are involved. For the ICP surface registration algorithm to be able to converge to a reasonable solution, the range scan must cover a large proportion of the anterior surface, as in the seven datasets. However when that condition is not true the computational algorithm would not perform well.

The discrepancy in the values of various parameters for the other dataset might be due to organ motion. The visual difference in the registration results can be seen in Figure 12 below.

(a)
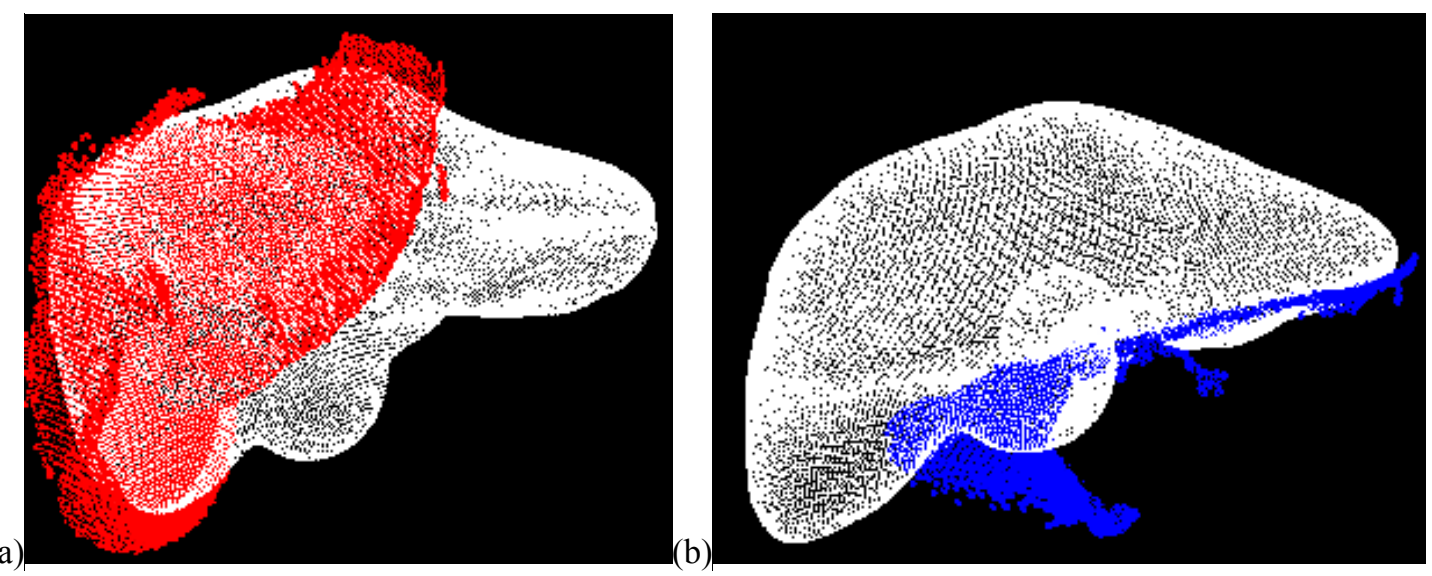

Figure 12: Results of registration using (a) computational method and (b) anatomical method. 
In this case the result from the anatomical landmarks method (Figure 12 (b)) shows gross misalignment. The LRS surface is registered to the inferior surface of the liver instead of the anterior surface. Since the results from the anatomical landmarks method were considered the "gold standard" for this study, the accuracy of the registration results for this dataset can not be evaluated.

In comparison of RMS residual distances in Figure 6 the values for the fifth dataset are unexpectedly higher than the other datasets. However, as seen in Figure 7 and Figure 8, both the methods yield similar transformations. The large RMS residual was explained by visual examination of the registration results as shown in Figure 13 below.

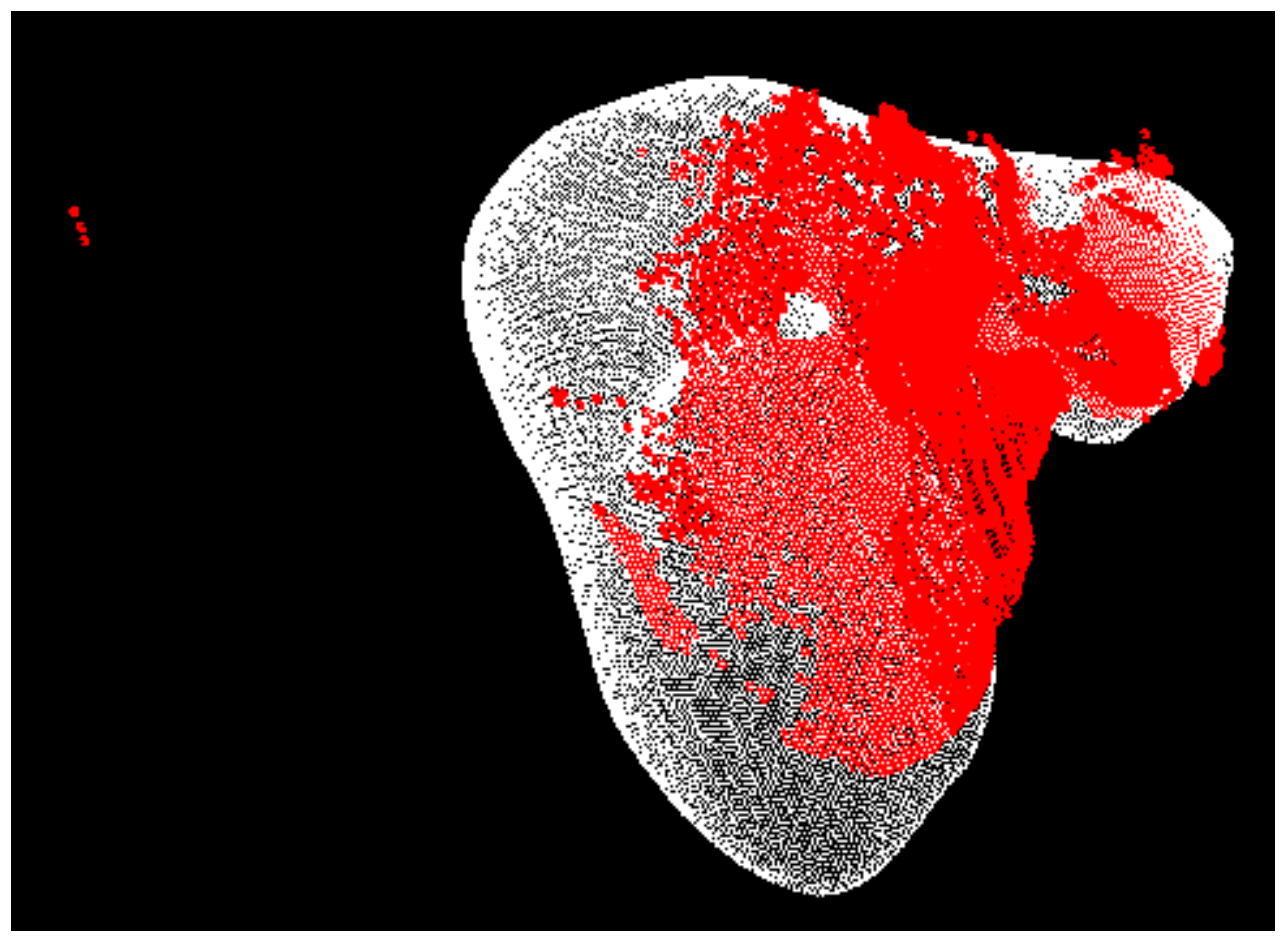

Figure 13: Registration results of the fifth dataset in Figure 6. Extraneous points due to inaccurate segmentation can be seen on extreme left of the image.

Inaccurate segmentation might be the reason for the large RMS residual value as some misclassified points (seen in the upper left in Figure 13) contributed to the error. 
The time taken by the ICP algorithm to find a solution using initial pose from the anatomical landmarks method was less than using the computational method for most cases, although as indicated by the t-test statistic, the time difference isn't statistically significant. Smaller run time for the anatomical method could potentially be explained due to better correspondence between the homologous points in the anatomical landmarks method. There are more approximations involved in finding the initial pose in the computational method, thus the pre-alignment for the ICP might be slightly better with the anatomical method; requiring fewer iterations for convergence to a solution. However the computational time presented in Error! Reference source not found. does not take into account the time required to find the homologous points to obtain the initial pose. The computational method only requires an approximate input of the anatomical orientation of the liver as opposed to the specific anatomical landmarks on the liver surface. Therefore in practice the computational method might take similar or less amount of time as the anatomical landmarks method.

Some retrospective studies were performed to study the effects of varying some parameters in the algorithm. The length of the computational cross (shown in Figure 3) was varied from $40 \%$ to $90 \%$ of the maximum lengths for computing the homologous points. The effect on the RMS residual distance, the translation vector and the Euler angles of rotation for datasets was observed (shown in Table 2). The standard deviations across that range of values indicate that the registration results are sensitive to the length of the computational cross. For most of the datasets, $70 \%$ of the length of the computational cross yielded results most similar to those using the anatomical landmarks method. Therefore the value of $70 \%$ was selected for this study as a satisfactory distance for the four homologous points. 
The results of the study to observe the angle between the vectors $\hat{x}_{p}^{\prime}$ and $\hat{x}_{p}$ for the CT data, and the analogous vectors in LRS data were shown in Table 3. On an average, this angle was significantly greater for the LRS data than the CT data. This might be because CT data is a complete representation of the liver surface and has a characteristic shape. Therefore the superior and the extreme left regions could be picked by the user with a greater consistency across the datasets. This might be harder in the LRS dataset, which is a partial representation of the data. It doesn't have a characteristic shape to provide cues about the orientation. However the mean angles for the seven datasets were not significantly lower than the mean angles for all datasets. Therefore this change in the angle could not be correlated to the bad registration results in the last two datasets. 


\section{CHAPTER V}

\section{CONCLUSIONS}

The computational method performed well in seven of the nine datasets that were

tested and evaluated using the results from the anatomical landmarks method as "gold standard". As seen in one of the datasets, for optimal performance, the computational method assumes the laser range scan covers a majority of the anterior surface of the liver. The mean computational time for the ICP algorithm was longer for the tested data using the initial pose obtained from the computational method as compared to the anatomical landmarks method. However it does not take into account the time required for localizing the anatomical landmarks. Thus the computational method, in practice, might not require a significantly longer time. Among other limitations, the registration results would be dependent on point selection by the user. Although the initial pose is calculated by approximations, but depending on the nature of the surface of an individual dataset, the amount of motion, as well as the user selection of the regions for anatomical orientation, the registration results might be inaccurate. Future studies would include testing the inter-user variability in region selection. It might also be possible to reduce the computation time by improvement in the approximations in the algorithm to provide a more exact initial pose, which would reduce the number of iterations required by the ICP algorithm to compute the solution. However, even with the above stated limitations the method presented in this manuscript is promising because results obtained with an approximated input of orientation were comparable to a method that requires precise localization of anatomical landmarks. The method has additional 
advantage of reducing contact between the probe and the resected liver, thus reducing OR time and potential deformation. 


\section{APPENDIX}

\section{EFFECT OF CHANGING THE LENGTH OF THE COMPUTATIONAL CROSS}

The results of the retrospective study of finding the effect of changing the length of the computational cross on the registration results are presented below. The proportion of the computational cross (shown in Figure 3) used for calculating the homologous points was varied from $40 \%$ of the length to $90 \%$ of the length, in $10 \%$ increments. The effect of this change was studied on the nine datasets, and the results are presented in the table below.

Table 4: Effect of changing the proportion of the lengths xmax and zmax for homologous point selection on the registration results of nine datasets.

\begin{tabular}{|c|c|c|c|c|c|c|c|c|}
\hline \multicolumn{9}{|c|}{ Dataset \#1 } \\
\hline $\begin{array}{c}\% \text { of } \\
\text { lengths }\end{array}$ & 40.00 & 50.00 & 60.00 & 70.00 & 80.00 & 90.00 & $\begin{array}{l}\text { Standard } \\
\text { Deviation }\end{array}$ & $\begin{array}{c}\text { Anatomical } \\
\text { Method }\end{array}$ \\
\hline $\begin{array}{c}\text { RMS } \\
\text { Residual }\end{array}$ & 4.16 & 4.16 & 4.16 & 4.16 & 4.16 & 4.16 & 0.00 & 4.16 \\
\hline$t_{x}$ & 44.53 & 45.36 & 108.41 & 125.72 & 2.92 & 41.11 & 46.31 & 126.30 \\
\hline$t_{y}$ & -216.65 & -226.65 & -372.03 & -388.26 & -363.36 & -377.22 & 79.77 & -388.15 \\
\hline$t_{z}$ & 495.26 & 484.17 & 260.81 & 191.33 & 203.49 & 163.35 & 150.58 & 192.25 \\
\hline$\theta_{x}$ & -13.10 & -13.47 & -48.49 & -72.47 & -57.40 & -80.38 & 28.79 & -72.27 \\
\hline$\theta_{y}$ & 48.57 & 50.04 & 71.84 & 74.10 & 77.79 & 76.18 & 13.42 & 73.98 \\
\hline$\theta_{z}$ & -114.42 & -114.26 & -138.72 & -160.41 & -157.45 & -176.45 & 25.65 & -160.16 \\
\hline \multicolumn{9}{|c|}{ Dataset \#2 } \\
\hline $\begin{array}{c}\% \text { of } \\
\text { lengths }\end{array}$ & 40.00 & 50.00 & 60.00 & 70.00 & 80.00 & 90.00 & $\begin{array}{l}\text { Standard } \\
\text { Deviation }\end{array}$ & $\begin{array}{l}\text { Anatomical } \\
\text { Method }\end{array}$ \\
\hline $\begin{array}{c}\text { RMS } \\
\text { Residual }\end{array}$ & 5.64 & 5.64 & 5.64 & 5.64 & 5.64 & 5.64 & 0.00 & 7.301932 \\
\hline$t_{x}$ & -319.60 & -315.97 & -298.87 & -288.45 & -301.76 & -330.91 & 15.61 & 33.26 \\
\hline$t_{y}$ & 4.50 & -9.26 & -6.01 & 7.72 & 3.28 & -33.93 & 15.33 & -332.81 \\
\hline $\mathrm{t}_{\mathrm{z}}$ & 390.74 & 384.70 & 407.87 & 428.65 & 409.43 & 331.15 & 33.64 & 216.73 \\
\hline$\theta_{\mathrm{x}}$ & 5.04 & 5.67 & 7.83 & 7.35 & 6.78 & -3.32 & 4.15 & 141.41 \\
\hline$\theta_{y}$ & 71.91 & 72.64 & 69.38 & 66.53 & 69.14 & 79.22 & 4.38 & 87.90 \\
\hline$\theta_{z}$ & -149.56 & -147.18 & -143.71 & -145.00 & -146.31 & -155.24 & 4.14 & 38.69 \\
\hline \multicolumn{9}{|c|}{ Dataset \#3 } \\
\hline $\begin{array}{c}\% \text { of } \\
\text { lengths }\end{array}$ & 40.00 & 50.00 & 60.00 & 70.00 & 80.00 & 90.00 & $\begin{array}{l}\text { Standard } \\
\text { Deviation }\end{array}$ & $\begin{array}{l}\text { Anatomical } \\
\text { Method }\end{array}$ \\
\hline $\begin{array}{c}\text { RMS } \\
\text { Residual }\end{array}$ & 3.84 & 3.84 & 3.84 & 3.84 & 3.84 & 3.84 & 0.00 & 3.844645 \\
\hline$t_{x}$ & -98.35 & -103.01 & -94.54 & -60.88 & -36.54 & -39.16 & 30.41 & -60.8622 \\
\hline$t_{y}$ & -256.73 & -254.39 & -257.87 & -269.26 & -273.74 & -267.76 & 7.96 & -269.275 \\
\hline$t_{z}$ & 47.18 & 63.12 & 67.59 & 30.23 & 5.30 & -12.01 & 31.95 & 30.2905 \\
\hline
\end{tabular}




\begin{tabular}{|c|c|c|c|c|c|c|c|c|}
\hline$\theta_{x}$ & -43.63 & -35.27 & -31.20 & -40.44 & -51.70 & -60.11 & 10.69 & -40.7742 \\
\hline$\theta_{y}$ & 73.65 & 72.40 & 72.10 & 76.06 & 78.16 & 79.59 & 3.12 & 76.0248 \\
\hline$\theta_{z}$ & -141.26 & -133.97 & -128.97 & -133.42 & -141.23 & -149.85 & 7.49 & -133.751 \\
\hline \multicolumn{9}{|c|}{ Dataset \#4 } \\
\hline $\begin{array}{c}\% \text { of } \\
\text { lengths }\end{array}$ & 40.00 & 50.00 & 60.00 & 70.00 & 80.00 & 90.00 & $\begin{array}{l}\text { Standard } \\
\text { Deviation }\end{array}$ & $\begin{array}{c}\text { Anatomical } \\
\text { Method }\end{array}$ \\
\hline $\begin{array}{c}\text { RMS } \\
\text { Residual }\end{array}$ & 7.82 & 7.82 & 7.82 & 7.82 & 7.82 & 7.82 & 0.00 & 4.43021 \\
\hline$t_{x}$ & -44.19 & -67.78 & -119.62 & -133.52 & -162.65 & -183.36 & 53.81 & 45.05 \\
\hline$t_{y}$ & -238.20 & -244.73 & -223.11 & -225.38 & -203.80 & -180.05 & 23.82 & 75.17 \\
\hline$t_{z}$ & 371.19 & 342.54 & 327.78 & 304.34 & 306.36 & 319.23 & 25.22 & 587.95 \\
\hline$\theta_{\mathrm{x}}$ & -53.53 & -57.84 & -62.09 & -67.33 & -66.80 & -64.73 & 5.44 & -10.00 \\
\hline$\theta_{y}$ & 57.60 & 60.68 & 59.61 & 59.40 & 60.06 & 58.36 & 1.13 & 27.46 \\
\hline$\theta_{z}$ & -178.80 & 176.34 & 165.61 & 159.43 & 156.16 & 153.92 & 139.48 & 179.75 \\
\hline \multicolumn{9}{|c|}{ Dataset \#5 } \\
\hline $\begin{array}{c}\% \text { of } \\
\text { lengths }\end{array}$ & 40.00 & 50.00 & 60.00 & 70.00 & 80.00 & 90.00 & $\begin{array}{l}\text { Standard } \\
\text { Deviation }\end{array}$ & $\begin{array}{c}\text { Anatomical } \\
\text { Method }\end{array}$ \\
\hline $\begin{array}{c}\text { RMS } \\
\text { Residual }\end{array}$ & 23.95 & 23.95 & 23.95 & 23.95 & 23.95 & 23.95 & 0.00 & 23.95257 \\
\hline$t_{x}$ & 279.87 & 283.26 & 9.50 & 2.13 & -5.35 & 13.67 & 142.97 & 3.0205 \\
\hline$t_{y}$ & -368.71 & -373.37 & -388.90 & -373.64 & -371.15 & -372.69 & 7.17 & -373.987 \\
\hline$t_{z}$ & 539.92 & 526.86 & 426.53 & 472.16 & 471.21 & 481.01 & 41.35 & 471.8185 \\
\hline$\theta_{\mathrm{x}}$ & 12.76 & 18.95 & 59.31 & 44.60 & 38.95 & 34.06 & 17.04 & 44.551 \\
\hline$\theta_{y}$ & 69.99 & 70.70 & 73.55 & 71.32 & 73.02 & 72.70 & 1.42 & 71.3734 \\
\hline$\theta_{z}$ & -60.77 & -53.93 & -42.25 & -57.47 & -64.27 & -67.08 & 8.87 & -57.45 \\
\hline \multicolumn{9}{|c|}{ Dataset \#6 } \\
\hline $\begin{array}{c}\% \text { of } \\
\text { lengths }\end{array}$ & 40.00 & 50.00 & 60.00 & 70.00 & 80.00 & 90.00 & $\begin{array}{l}\text { Standard } \\
\text { Deviation }\end{array}$ & $\begin{array}{l}\text { Anatomical } \\
\text { Method }\end{array}$ \\
\hline $\begin{array}{c}\text { RMS } \\
\text { Residual }\end{array}$ & 6.56 & 6.56 & 6.56 & 6.56 & 6.56 & 6.56 & 0.00 & 6.5602 \\
\hline$t_{x}$ & 1.09 & 25.73 & 10.06 & -0.08 & 59.26 & 32.90 & 22.75 & -0.1035 \\
\hline$t_{y}$ & -258.45 & -265.88 & -269.14 & -274.31 & -279.93 & -285.95 & 9.91 & -274.298 \\
\hline$t_{z}$ & 380.07 & 376.88 & 364.33 & 352.69 & 361.06 & 345.47 & 13.43 & 352.6748 \\
\hline$\theta_{x}$ & 82.40 & 84.42 & 92.52 & 99.02 & 95.27 & 102.69 & 8.01 & 99.0324 \\
\hline$\theta_{y}$ & 79.98 & 79.88 & 78.50 & 77.30 & 79.53 & 77.15 & 1.27 & 77.3083 \\
\hline$\theta_{z}$ & -20.58 & -15.14 & -8.36 & -2.68 & 0.32 & 5.21 & 9.73 & -2.6723 \\
\hline \multicolumn{9}{|c|}{ Dataset \#7 } \\
\hline $\begin{array}{c}\% \text { of } \\
\text { lengths }\end{array}$ & 40.00 & 50.00 & 60.00 & 70.00 & 80.00 & 90.00 & $\begin{array}{l}\text { Standard } \\
\text { Deviation }\end{array}$ & $\begin{array}{c}\text { Anatomical } \\
\text { Method }\end{array}$ \\
\hline $\begin{array}{c}\text { RMS } \\
\text { Residual }\end{array}$ & 2.77 & 2.77 & 2.77 & 2.77 & 2.77 & 2.77 & 0.00 & 2.770235 \\
\hline$t_{x}$ & -142.27 & -145.39 & -197.18 & -169.23 & -277.77 & -240.89 & 54.53 & -163.078 \\
\hline$t_{y}$ & -254.44 & -235.80 & -185.17 & -261.13 & -221.29 & -253.62 & 28.56 & -265.287 \\
\hline$t_{z}$ & 415.23 & 440.70 & 456.64 & 369.06 & 220.85 & 179.54 & 118.24 & 368.6508 \\
\hline$\theta_{\mathrm{x}}$ & 18.40 & 19.07 & 14.28 & 36.23 & 168.86 & 178.99 & 78.88 & 35.6796 \\
\hline$\theta_{y}$ & 76.29 & 72.67 & 71.09 & 79.81 & 81.54 & 77.05 & 4.02 & 79.9884 \\
\hline$\theta_{z}$ & -92.51 & -92.28 & -105.89 & -76.74 & 42.15 & 57.29 & 73.85 & -76.5909 \\
\hline \multicolumn{9}{|c|}{ Dataset \#8 } \\
\hline $\begin{array}{c}\% \text { of } \\
\text { lengths }\end{array}$ & 40.00 & 50.00 & 60.00 & 70.00 & 80.00 & 90.00 & $\begin{array}{l}\text { Standard } \\
\text { Deviation }\end{array}$ & $\begin{array}{c}\text { Anatomical } \\
\text { Method }\end{array}$ \\
\hline $\begin{array}{c}\text { RMS } \\
\text { Residual }\end{array}$ & 5.24 & 5.24 & 5.24 & 5.24 & 5.24 & 5.24 & 0.00 & 5.244178 \\
\hline$t_{x}$ & -122.82 & -125.78 & -99.08 & 35.74 & 6.40 & -104.74 & 70.65 & 35.7033 \\
\hline$t_{y}$ & -85.16 & -70.80 & -73.39 & -108.50 & -69.88 & -228.93 & 61.90 & -108.515 \\
\hline
\end{tabular}




\begin{tabular}{|c|c|c|c|c|c|c|c|c|}
\hline $\mathrm{t}_{z}$ & 519.60 & 525.99 & 539.63 & 560.88 & 579.08 & 311.36 & 97.93 & 560.8574 \\
\hline$\theta_{x}$ & 1.69 & 1.17 & -0.82 & -10.85 & -8.14 & 1.29 & 5.47 & -10.8507 \\
\hline$\theta_{y}$ & 47.93 & 46.91 & 44.83 & 40.65 & 37.44 & 75.30 & 13.55 & 40.6555 \\
\hline$\theta_{z}$ & -120.20 & -122.53 & -121.56 & -113.40 & -116.95 & -110.22 & 4.88 & -113.396 \\
\hline \multicolumn{7}{|c|}{ Dataset \#9 } \\
\hline $\begin{array}{c}\% \text { of } \\
\text { lengths }\end{array}$ & 40.00 & 50.00 & 60.00 & 70.00 & 80.00 & 90.00 & $\begin{array}{c}\text { Standard } \\
\text { Deviation }\end{array}$ & $\begin{array}{c}\text { Anatomical } \\
\text { Method }\end{array}$ \\
\hline $\begin{array}{c}\text { RMS } \\
\text { Residual }\end{array}$ & 5.37 & 5.37 & 5.37 & 5.37 & 5.37 & 5.37 & 0.00 & 5.372512 \\
\hline$t_{x}$ & 47.51 & -14.87 & -52.52 & 143.83 & 125.36 & 131.87 & 83.44 & 143.8773 \\
\hline$t_{y}$ & -299.98 & -308.76 & -295.59 & -314.43 & -306.35 & -305.51 & 6.61 & -314.436 \\
\hline$t_{z}$ & 16.01 & 179.53 & 168.34 & 292.62 & 312.40 & 307.68 & 115.85 & 292.6879 \\
\hline$\theta_{x}$ & 175.96 & 175.46 & -177.94 & -114.24 & -97.36 & -101.29 & 156.82 & -114.183 \\
\hline$\theta_{y}$ & 55.68 & 74.86 & 73.40 & 82.39 & 83.32 & 82.67 & 10.56 & 82.4143 \\
\hline$\theta_{z}$ & 80.53 & 73.50 & 75.37 & 159.13 & 173.88 & 170.70 & 50.37 & 159.1851 \\
\hline
\end{tabular}




\section{REFERENCES}

1. R.L. Galloway, "The process and development of image-guided procedures," Ann. Rev. Biomed. Eng., 3, 83-108, 2001

2. A.J. Herline, J.D. Stefansic, J.P. Debelak, S.L. Hartmann, C.W. Pinson, R.L. Galloway, and W.C. Chapman, "Image guided surgery: preliminary feasibility studies of frameless stereotactic liver surgery," Arch. Surg., 134, 644-649, 1999.

3. J.D. Engle and L.D. Lunsford, "Brain tumor resection guided by intraoperative computed tomography," J. Neuro-Oncol., 4, 361-370, 1987.

4. C. Nimsky, O. Ganslandt, H. Kober, M. Buchfelder, and R. Fahlbusch, "Intraoperative magnetic resonance imaging combined with neuronavigation: a new concept," Neurosurgery, 48, 1082-1089, 2001.

5. R.M. Comeau, A.F. Sadikot, A. Fenster, and T.M. Peters, "'Intraoperative ultrasound for guidance and tissue shift correction in image-guided neurosurgery," Med. Phys. 27, 787-800, 2000.

6. A.J. Herline, J.L. Herring, J.D. Stefansic, W.C. Chapman, R.L. Galloway, and B.M. Dawant, "Surface registration for use in interactive, image-guided liver surgery," Comput. Aided Surg., 5, 11-17, 2000

7. D.M. Cash, T.K. Sinha, W.E. Chapman, R.L. Galloway, and M.I. Miga, "Fast, accurate surface acquisition using a laser range scanner for image-guided liver surgery," Proc. SPIE Int. Soc. Opt. Eng., 4681, 100-110, 2002.

8. D.M. Cash, W.C. Chapman, H. Terawaki, B.M. Dawant, R.L. Galloway, and M.I. Miga. "Incorporation of a laser range scanner into image guided liver surgery:

Surface acquisition, registration and tracking." Med. Phys. 30, 1671-1682, July 2003

9. C.A. Pelizzari, G.T. Chen, D.R. Spelbring, R.R. Weichselbaum, and C.T. Chen, "Accuarate three-dimensional registration of CT, PET, and/or MR images of the brain," J Comput. Assist. Tomogr., 13, 20-26, 1989.

10. D.L.G. Hill, C. Studholme, and D.J. Hawkes, "Voxel similarity measures for automated image registration,” Proc. SPIE Int. Soc. Opt. Eng., 2359, 205-216, 1994.

11. F. Mael, A. Collignon, D. Vandermeulen, G. Marchal, and P. Suetens, "Multimodality image registration by maximization of mutual information," IEEE Trans. Med. Imaging, 16(2), 187-198, 1997.

12. W.M. Wells, III, P. Viola, H. Atsumi, S. Nakajima, and R. Kikinis, "Multi-modal volume registration by maximization of mutual information," Med. Image Anal., 1(1), 35-51, 1996. 
13. J.B. West, J.M. Fitzpatrick, M.Y. Wang, B.M. Dawant, C.R. Maurer, Jr., R.M. Kessler, R.J. Maciunas, C. Barillot, D. Lemoine, A. Collignon, F. Maes, P. Suetens, D. Vandermeulen, P.A. van den Elsen, S. Napel, T.S. Sumanaweera, B. Harkness, P.F. Hemler, D.L.G. Hill, D.J. Hawkes, C. Studholme, J.B.A. Maintz, M.A. Viergever, G. Malandain, X. Pennec, M.E. Noz, G.Q. Maguire, Jr., M. Pollack, C.A. Pelizzari, R.A. Robb, D. Hanson, and R.P. Woods, "Comparison and evaluation of retrospective intermodality brain image registration techniques," J. Comput. Assist. Tomogr., 21, 554-566, 1997.

14. P.J. Besl and N.D. McKay, "A method for registration of 3-D shapes," IEEE Trans. Pattern Anal. Mach. Intell., 14, 239-256, 1992.

15. K.S. Arun, T.S. Huang, and S.D. Blostein, "Least square fitting of two 3-D point sets," IEEE Trans. Pattern Anal. Mach. Intell., 9, 698-700, 1987.

16. T.L. Faber and EM Stokely, "Orientation of 3-D structures in medical images," IEEE Trans Pat Anal and Mach Intel, 10, 626-633, 1988.

17. N.M. Alpert, J.F. Bradshaw, D. Kennedy, and J.A. Correia, "The principal axis transformation- a method for image registration", J Nucl Med, 31, 1717-1722, 1990.

18. H. Rusinek, W. Tsui, A.V. Levy, M.E. Noz, and M.J. de Leon, "Principal axes and surface fitting methods for three-dimensional image registration," J Nucl Med, 34, 2019-2024, 1993.

19. L.W. Clements, D.M. Cash, T.K. Sinha, R.L. Galloway, "Semiautomatic segmentation of textured laser range scans for use in image-guided procedures," Proc. SPIE Int. Soc. Opt. Eng., 5747, 1658-66, 2005

20. M.Y. Wang, J.M. Fitzpatrick, and C.R. Maurer, Jr., "Design of fiducials for accurate registration of CT and MR volume images," Proc. SPIE Int. Soc. Opt. Eng., 2434, 96$108,1995$.

21. L. W. Clements, D. M. Cash, W. C. Chapman, R. L. Galloway, and M. I. Miga, "Robust surface registration using salient anatomical features in image-guided liver surgery", Proc. SPIE Int. Soc. Opt. Eng., 2006

22. C. R. Maurer, G. B. Aboutanos, B. M. Dawant, R. J. Maciunas, and J. M. Fitzpatrick, "Registration of 3-D images using weighted geometrical features," IEEE Trans. Med. Imaging, 15, 836-849, 1996.

23. D. M. Cash, M. I. Miga, T. K. Sinha, R. L. Galloway, and W. C. Chapman, "Compensating for intraoperative soft-tissue deformations using incomplete surface data and finite elements," IEEE Trans. Med. Imaging, 24, 1479-1491, 2005. 